Frequency as a Barrier to Learning Complex Nominal Morphology

\author{
Sara Finley \\ Pacific Lutheran University \\ finleysr@plu.edu
}

\title{
Acknowledgments
}

I would like to thank anonymous reviewers for their helpful feedback on previous versions of this paper.

I am also grateful for Jeffrey Parker, Vsevolod Kapatsinski, and other attendees of the 2017 Morphological Typology and Linguistic Cognition Workshop for thoughtful discussion. I am particularly grateful for Elizabeth Wiemers, Amanda-Fretland-Thomas, Paulin Schmidt, Saara Charania, Tiarra Lewis, Shelan Porter, Liza Radford, and Barbara Millward who provided help in developing stimuli, experimental design, implementation, and data collection. I am grateful to the Benson-Starkovich Faculty Development Award at Pacific Lutheran for providing financial support. I assume responsibility for any and all errors. 


\begin{abstract}
Most languages with highly structed morphological systems show some degree of syncretism, where the same affix is used for multiple categories. The typology of syncretism has suggested that syncretism is most likely to occur for structurally and semantically marked categories, but are often confounded with frequency. In two artificial grammar learning experiments, English-speaking adults were exposed to a 3-gender x 3-number nominal system, where one number category (Singular, Dual, or Plural) showed syncretism across gender. In the experiment, the frequency of the syncretic morpheme was equal to non-syncretic morphemes, but there were $3 \mathrm{x}$ fewer items containing the syncretic morpheme. In Experiment 1, participants failed to learn the syncretic morpheme, with no biases for marked categories. In Experiment 2, when the low frequency category was always neuter, participants learned the category in all cases except the dual. These results suggest that low frequency of syncretic items significantly impairs learning syncretic categories. Suggestions for design and implementation of learning low frequency categories are discussed.
\end{abstract}

Keywords: artificial grammar learning; morphology; learning biases; syncretism 


\section{Frequency as a Barrier to Learning Complex Nominal Morphology}

Many languages have complex morphological systems, where learners must discover how form and meaning relate to each other, despite a lack of exposure to all forms in a paradigm (Harmon \& Kapatsinski, 2017). For example, in a language where the nominal system has three genders and three numbers, each noun might have one of nine morphological markers. One possible route to decreasing the complexity of the morphological system is through syncretism. In syncretism, the same form is applied to several morphemes. In Russian, for example, the dative plural suffix /-am/ applies to all four noun classes, while the instrumental singular affix /-oj/ applies only to Class II nouns (Parker, 2016).

While syncretism could decrease the number of forms that are required to be learned, informational load from ambiguity is also increased, as the same form applies has several different meanings. Previous researched has shown that the learnability of syncretic forms improves with reliability (e.g., if the syncretism applies across a similar category) (Finley \& Wiemers, 2015; Pertsova, 2011), suggesting that syncretism may decrease the cognitive load of the learning problem when there is a semantic connection between syncretic items.

Another possible way that syncretism could help decrease the learning problem is to increase the frequency of marked or infrequent items. The dual gender is considered to be both semantically and cognitively marked (Nevins, 2011). On its own, learning the dual neuter (for example) may be harder to learn than the singular neuter form simply because the input contains more singular neuter forms (Parker, 2016). If the dual neuter shares the same form as, for example, the dual masculine, then the frequency of these categories could 'gang' up, and increase the frequency of the marked category, thereby increasing its learnability. In this case, the language learner might be exposed to the same number of words with the singular neuter as the entire dual 
category, but because there is only one form to learn, the language learner may show no deficits for learning the less frequent, semantically marked category.

Research in the typology of syncretism has suggested that marked categories are more likely to show syncretism (Baerman et al., 2005; Müller, 2007). Many languages (including English) collapse the dual with the plural, and many languages with gender system show syncretism for non-singular numbers (Corbett, 1991). One question is why these typological trends occur. On the one hand, there is a link between markedness and syncretism, whereby more marked categories are more likely show neutralization (Aalberse, 2009; Nevins, 2006). However, markedness is a loaded term (Haspelmath, 2006), which confounds frequency and complexity 1 (as well as other factors). It is possible that the typological trends for syncretism for marked categories like dual is such because dual is low frequency, rather than any inherent complexity such as semantic or structural markedness in the dual. The artificial language learning paradigm can be used to tease these factors apart because it is possible to specifically control for frequency of items in the training set.

Previous research using the artificial language learning paradigm has shown that learners are biased towards syncretism in semantically/featurally marked categories (Finley, in press; Lee, 2020). Lee (2020) showed improved learning for dual-plural syncretism, but worse learning for singular-dual and singular-plural syncretism. Finley (in press) showed an increased benefit for syncretism when syncretism across gender applied to the dual category. However, in Finley's experiments, the number of items from each semantic category was equal, meaning that the syncretic morphemes were more frequent than the non-syncretic morphemes. In those experiments, the syncretic categories were always learned better than non-syncretic categories, likely due to frequency. In the present study, each form appeared the same number of times, thus 
reducing the overall frequency of items from the syncretic categories. If syncretism allows for learnability of infrequent categories, then participants should learn the syncretic categories, despite the lower frequency. If learnability of syncretic categories is dependent on markedness, then participants should be more likely to learn the syncretic category for marked (dual) items.

\section{Experiment 1}

Experiment 1 tested for the role of marked categories and frequency in the learnability of noun categories with syncretism. Participants were exposed to a miniature language with a 3 (gender) by 3 (number) system that was syncretic for gender across the three numbers (singular, dual, and plural). In the present experiment, the number of items ending in each morpheme was identical for all morphemes $(\mathrm{n}=6)$, but the morpheme for the syncretic category $(/-\mathrm{go} /)$ represented three different categories (masculine, feminine, and plural).

\section{Method}

\section{Participants}

All participants in Experiment 1 were adult, English speakers, recruited from the Psychology subject pool at a university in the Pacific Northwest of the United States, and were given course credit for their participation. Final data analysis included 68 participants ( 22 in the Dual condition, and 23 each in the Plural and Singular conditions). An additional 10 participants were run but excluded from analyses: two failed to complete the study in less than 45 minutes, and eight indicated (in a post-completion survey) that they wished their data to be discarded. All participants completed an online consent form for anonymous online surveys/experiments.

\section{Design and Materials}

Participants were auditorily exposed to a miniature, artificial language that contained CVCV stems (denoted by animals) with -CV suffixes denoting the gender ('masculine', 
'feminine', and 'neuter') and number (singular, dual, and plural) of the lexical item. While there were nine possible affixes, the language only contained seven, due to syncretism, which varied by condition. For example, all singular items took the suffix /-go/ regardless of gender in the Singular condition, and likewise for the dual in the Dual condition, and the plural in the Plural condition. All affix-category pairings are shown in Table 1. Copies of all stimuli files, data, and analysis code can be found at: https://osf.io/au5eb/.

The distinctions between gender and number were displayed using semantic markers on different animals. Number was indicated by the number of the animals in the picture display: one, two, or more than two. Gender was indicated using stereotyped accessories: bowtie for masculine, purse for feminine, and no accessories for neuter. Note that because stereotyped accessories were not transparent (e.g., some participants interpreted the bowtie as a hair bow), the gender system is ambiguous between an abstract grammatical gender system, rather than marking strict biosocial gender. While many languages show a correlation between grammatical gender and biosocial gender, many languages show noun class genders with no such distinction (Corbett, 2012).

Table 1: Gender and Number Markings

\begin{tabular}{llllllllll}
\hline Cond & \multicolumn{3}{c}{ Syncretism in Singular } & \multicolumn{3}{c}{ Syncretism in Dual } & \multicolumn{3}{c}{ Syncretism in Plural } \\
& 1 & 2 & Pl & 1 & 2 & Pl & 1 & 2 & Pl \\
& & & & & & & & & \\
\hline Masc & go & me & ge & me & go & ge & me & ge & go \\
Fem & go & di & po & di & go & po & di & po & go \\
Neuter & go & wa & mi & wa & go & mi & wa & mi & go \\
\hline
\end{tabular}

Each training trial consisted of a picture of one of 12 animal stems varying by gender and number (e.g., one penguin with a purse), and a sound file to denote the meaning of the word. The CVCV$\mathrm{CV}$ words contained a mix of consonants from the set $[\mathrm{b}, \mathrm{d}, \mathrm{g}, \mathrm{k}, \mathrm{m}, \mathrm{n}, \mathrm{p}, \mathrm{s}, \mathrm{t}, \mathrm{v}, \mathrm{w}, \mathrm{z}]$ and a mix of vowels from the set [a, e, i, o, u]. No item shared a close resemblance to known English words. 
Examples of the picture-sound pairings can be found in Table 2. Note that the images were resized to fit the tables in the paper.

Table 2: Example Training Trials (Singular Condition)

\begin{tabular}{l}
\hline Meaning/Sound \\
Singular-Fem \\
"satego" \\
Plural-Neuter \\
"pazimi" \\
$\begin{array}{l}\text { Dual-Masculine } \\
\text { "tiseme" }\end{array}$
\end{tabular}

The language contained 12 stems, and 9 suffixes, for a total of 108 possible words in the language. Participants were exposed to a subset (half) of these words in training, and then tested on novel items. Each participant was exposed to six forms for each of the seven suffixes for a total of 42 (6x7) items. If all possible genders and numbers were presented an equal number of times, it would mean the syncretic allomorph would be heard three times more than all other morphemes, but in three different semantic contexts (as was done in Finley, in press). In the present study, the semantic contexts were heard less frequently to allow for an even distribution of the forms.

Learnability of the paradigm was assessed via a two-alternative forced choice task. The task required participants to select the best (audio) form for the given (picture) meaning. A single picture was shown, and two different forms were presented, both with the same stem, but a different ending. This ensured that that the participant relied on knowledge of the suffixes to determine the correct response. The incorrect response was always the same suffix for the same gender, but a different number. The incorrect (foil) response was split evenly between the two other possible numbers, where possible. For example, if the target picture contained two male 
ladybugs, the incorrect response would be the word for either one male ladybug, or plural male ladybugs. Controlling for gender in this way makes it possible to test the learnability of the various numbers. Number was tested (as opposed to gender) because the hypothesis in the experiment related most specifically to the learnability of the various numbers. Because the genders used in this experiment were ambiguous between noun class genders rather than biosocial genders, there were no specific hypotheses about the learnability of gender. While masculine gender tends to be the least marked gender (Corbett, 2012), neuter may be less marked in this experiment because neuter items were plain animals. Examples of test items can be found in Table 3.

Table 3: Example Test Trials (Singular Condition)

\begin{tabular}{lll}
\hline Correct Item & Foil Item & Picture \\
\hline vopidi & vopipo
\end{tabular}

\section{Procedure}

The experiment was conducted online using FindingFive (FindingFive Team, 2019). Participants were instructed to complete the entire experiment in one sitting, using headphones, and in a quiet location with stable internet connection. Participants were asked to check their internet connection and audio using a test sound ([udvu]).

Participants were told that they were learning a novel language, and would be hearing words from the language, with a picture that denoted the meaning of the word. Participants were 
required to press a 'Continue' button after each sound file played. Following training, participants were encouraged to take a short break, and continue to the test phase. Participants were asked to pick which of two words best matched the meaning of the picture, the first or the second, and were given options to click on the corresponding choices or to press the 'a' key to indicate the first word, and the ' 1 ' key to indicate the second word. The audio and image files were always presented simultaneously.

Upon completion of the experiment, participants were given a written debriefing form, as well as a chance to place any feedback, and to recuse themselves from inclusion in data analysis. The entire experiment took approximately 20 minutes to complete.

\section{Results}

Means and standard errors of the mean for Experiment 1 can be found in Figure 1. Data from all eligible participants were included, but any trial that lasted longer than 10s were dropped $(\mathrm{n}=70)$. The data were fit into generalized linear mixed effect models fit by the Laplace approximation using the lme4 (Bates et al., 2015) package in R (R Development Core Team, 2018) via R Studio (RStudio Team, 2020). In order to test whether participants learned the syncretic pattern, a separate model was run for each training condition, with the condition and the syncretic test items set as the baseline (e.g., singular items in the Singular condition), with a significant intercept considered to be significantly different from chance, and therefore evidence of learning. All models included the maximal model that would converge (at minimum random intercepts for subjects and items). Redundant interaction comparisons are not reported. 


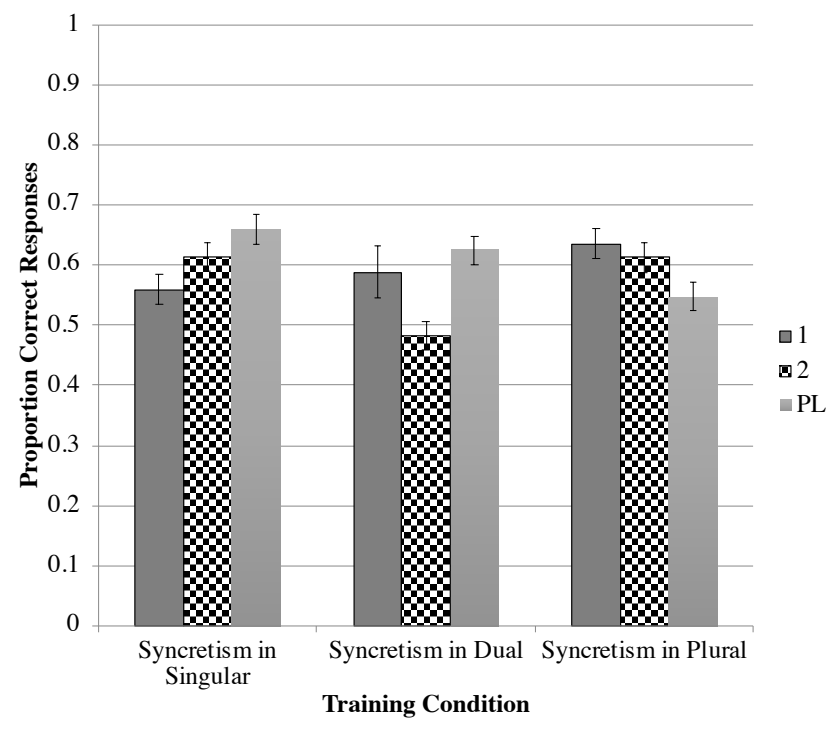

Figure 1: Experiment 1 Results.

\section{Dual Condition}

The model for the Dual condition did not show a significant intercept for dual (2) items, $\beta$ $=0.038, \mathrm{SE}=0.22, \mathrm{z}=-0.17, p=0.87$, suggesting that participants did not learn the dual form. Participants in the Dual condition were significantly more likely to select the correct response to singular (1) items $\beta=0.46, \mathrm{SE}=0.16, \mathrm{z}=2.89, p=0.0044$ and plural (PL) items $\beta=0.64, \mathrm{SE}=$ $0.16, \mathrm{z}=3.81, p<0.001$, compared to dual (2) items. There was a significant number by condition interaction for the difference between singular (1) and dual (2) items for the Dual condition compared to the Singular condition, $\beta=0.70, \mathrm{SE}=0.26, \mathrm{z}=2.75, p=0.0060$, but not for Dual condition compared the Plural condition, $\beta=0.25, \mathrm{SE}=0.23, \mathrm{z}=1.12, p=0.26$. There was a significant number by condition interaction for the difference between the dual (2) and plural (PL) items in the Plural condition compared to the Dual condition, $\beta=0.83, \mathrm{SE}=0.23, \mathrm{z}=3.66, p<$ 0.001 , but not the Singular condition compared to the Dual condition, $\beta=0.33$, SE $=0.26, \mathrm{z}=$ $1.26, p=0.21$.

\section{Plural Condition}


The model for the Plural condition did not show a significant intercept for plural (PL) items, $\beta=0.27, \mathrm{SE}=0.21, \mathrm{z}=1.23, p=0.22$, suggesting that participants did not learn the plural form. Participants in the Plural condition were significantly more likely to select the correct response to singular (1) items $\beta=0.40, \mathrm{SE}=0.16, \mathrm{z}=2.53, p=0.011$, but not dual (2) items $\beta=$ $0.19, \mathrm{SE}=0.16, \mathrm{z}=1.20, p=0.23$, compared to plural (PL) items. There were also significantly more correct responses plural (PL) items in the Singular condition, $\beta=0.40, \mathrm{SE}=0.16, \mathrm{z}=2.53$, $p=0.011$, but not the Dual condition, $\beta=0.19, \mathrm{SE}=0.16, \mathrm{z}=1.20, p=0.23$. There was a significant condition by number interaction for the difference between plural (PL) and dual (2) items for the Singular condition compared to the Plural condition, $\beta=0.49, \mathrm{SE}=0.22, \mathrm{z}=2.27, p$ $=0.023$. There were also significant condition by number interactions for the difference between plural (PL) and singular (1) items for both comparisons between Singular and Plural, $\beta=0.94$, SE $=0.22, \mathrm{z}=4.31, p<0.001$, and the Dual and Plural conditions, $\beta=0.57, \mathrm{SE}=0.21, \mathrm{z}=2.67, p=$ 0.0077 .

\section{Singular Condition}

The model for the Singular condition did not show a significant intercept for singular (1) items, $\beta=0.36, \mathrm{SE}=0.22, \mathrm{z}=1.65, p=0.10$, suggesting that participants did not learn the singular

form. Participants in the Singular condition were significantly more likely to select the correct response to plural (PL) items $\beta=0.54, \mathrm{SE}=0.16, \mathrm{z}=3.31, p<0.001$, but not dual (2) items $\beta=$ 0.23, $\mathrm{SE}=0.16, \mathrm{z}=1.46, p=0.16$, compared to singular (1) items. There were no significant differences in responses to singular (1) items between the Singular condition and either the Dual condition, $\beta=0.067, \mathrm{SE}=0.31, \mathrm{z}=0.22, p=0.83$, or the Plural condition, $\beta=0.31, \mathrm{SE}=0.30, \mathrm{z}$ $=1.01, p=0.31$. There was a significant condition by number interaction for the difference between singular (1) and dual (2) items for the Plural condition compared to the Singular condition, 
$\beta=0.44, \mathrm{SE}=0.22, \mathrm{z}=2.06, p=0.040$. The number by condition interaction for the difference between the difference between plural (PL) and singular (1) items was not significantly different between the Singular and the Dual conditions, $\beta=0.36, \mathrm{SE}=0.22, \mathrm{z}=1.66, p=0.10$.

Participants did not select the correct option to the syncretic categories at a rate significantly above chance for any condition, suggesting that low frequency of the syncretic items in this experiment significantly hindered learning. However, it is possible that participants made systematic assumptions about the syncretic categories. For example, since English does not have a dual morpheme, participants could have inferred that the dual and the plural were syncretic with each other. To test this, the data for the syncretic trials were compared for the two alternatives in exploratory models. In the Singular condition, participants chose the correct response to singular (1) items 62 percent of the time when the decoy item was plural (PL), and 52 percent of the time when the foil item was dual (2), but difference was only marginally significant, $\beta=0.51, \mathrm{SE}=$ $0.27, \mathrm{z}=1.86, p=0.062$. In the Dual condition, participants chose the correct response to dual (2) items 52 percent of the time when the decoy item was plural (PL), and 46 percent of the time when the foil item was singular (1); this difference was not significant, $\beta=0.14, \mathrm{SE}=0.23, \mathrm{z}=0.59, p$ $=0.55$. In the Plural condition, participants chose the correct response to plural (PL) items 56 percent of the time when the foil item was singular (1), and 53 percent of the time when the decoy item was dual (2); this difference was not significant, $\beta=0.28, \mathrm{SE}=0.23, \mathrm{z}=1.20, p=0.23$.

\section{Discussion}

The results of Experiment 1 showed that when the number of training items with the syncretic form for the entire categories was the same as the number of forms for individual nonsyncretic categories, and thus $3 x$ less, participants failed to learn the syncretic category. There did not seem to be a bias for or against learning the syncretic pattern for dual, singular or plural. 
Participants appeared to learn the non-syncretic forms, even though there were only six of forms of each morpheme, suggesting that learning morphological patterns requires a significant number of each individual category. One question that remains is whether the failure to learn the syncretic category in Experiment 1 was due the overall low frequency of the category (e.g., of singular items in the Singular condition), or of low frequency of individual category items (e.g., of singular neuter items in the Singular condition). To test this possibility, a second experiment was run in which the 'syncretic' condition had the same low frequency, but all items were neuter, holding out masculine and feminine items. This meant that the /-go/ items were the same frequency as all the other categories. In addition, participants were tested on the held-out items to probe how participants interpreted the missing items in the data, whether they assumed number to be syncretic across gender (e.g., all singular items in the Singular condition should have the same affix) or syncretic across number (e.g., masculine items collapsed across singular and plural).

Experiment 2 tested whether learnability of syncretic forms would increase when the training set did not include masculine or feminine items for the 'syncretic' condition. In the 'syncretic' condition, there were only neuter items (e.g., six neuter singular items ending in /-go/ in the Singular condition). In this case, it was impossible to know what the feminine and masculine forms for these items were. At test, participants were given a two-alternative forced choice, where the two options for held-out items were either/-go/, the same ending as the neuter, or the same ending as the other numbers. For example, in the Plural condition, the test item for feminine plural would have the options between /-go/ and either the feminine singular or the feminine dual. Thus, the held-out items tested participants preference/inference about whether syncretism applied to gender or to number. If participants select the /-go/ option, they assume that syncretism applies across gender. If participants select the other option, they assume that syncretism applies across number. 


\section{Experiment 2}

The present experiment tested for the role of marked categories and frequency in the learnability of noun categories with syncretism. Participants were exposed to a miniature language with a 3 (gender) by 3 (number) system that was syncretic for gender across the three numbers (singular, dual, and plural). In Experiment 1, the number of items ending in each morpheme was identical for all morphemes $(\mathrm{n}=6)$, but the morpheme for the syncretic category $(/$-go/) represented three different categories (masculine, feminine, and plural).

\section{Method}

\section{Participants}

All participants were adult, English speakers, recruited from the Psychology subject pool at a university in the Pacific Northwest of the United States, and did not participate in Experiment 1. Final data analysis included 56 participants (19 each in the Dual and Singular conditions, and 18 in the Plural condition). An additional four participants were run but included in the analyses: two were removed because they failed to complete the study in less than 45 minutes, and two were removed because they indicated in a post-completion survey that they wished their data to be discarded. No participant had previously participated in a similar study.

\section{Design and Materials}

The design and materials for Experiment 2 were similar to those of Experiment 1. The major difference was that rather than have syncretism in the infrequent category, the infrequent category only had neuter items, and there were the same number of these items as the other conditions. Thus, because the language no longer displays syncretism, the conditions are referred to as the Singular Hold-Out condition, where masculine and feminine items are held out in the singular, the Plural Hold-Out condition, where masculine and feminine items are held out in the 
plural, and the Dual Hold-Out condition, where masculine and feminine items are held out in the dual. Table 4 shows the design of Experiment 2.

Table 1: Gender and Number Markings (Experiment 2)

\begin{tabular}{|c|c|c|c|c|c|c|c|c|c|}
\hline \multirow[t]{2}{*}{ Cond } & \multicolumn{3}{|c|}{ Syncretism in Singular } & \multicolumn{3}{|c|}{ Syncretism in Dual } & \multicolumn{3}{|c|}{ Syncretism in Plural } \\
\hline & 1 & 2 & $\mathrm{P} 1$ & 1 & 2 & $\mathrm{Pl}$ & 1 & 2 & $\mathrm{Pl}$ \\
\hline Masc & & $\mathrm{me}$ & ge & $\mathrm{me}$ & & ge & me & ge & \\
\hline Fem & & di & po & di & & po & di & po & \\
\hline Neuter & go & wa & $\mathrm{mi}$ & wa & go & $\mathrm{mi}$ & wa & $\mathrm{mi}$ & go \\
\hline
\end{tabular}

\section{Procedure}

Experiment 2 utilized the same procedure as Experiment 1.

\section{Results}

The data were analyzed in a similar manner to Experiment 1, except that because Experiment 2 contained affixes that were not heard in training, these 'held out' affixes were analyzed separately. Means and standard errors of the mean for familiar/presented affixes can be found in Figure 2. Data from all eligible participants were included, but any trial that lasted longer than 10 s were dropped $(n=16)$. 


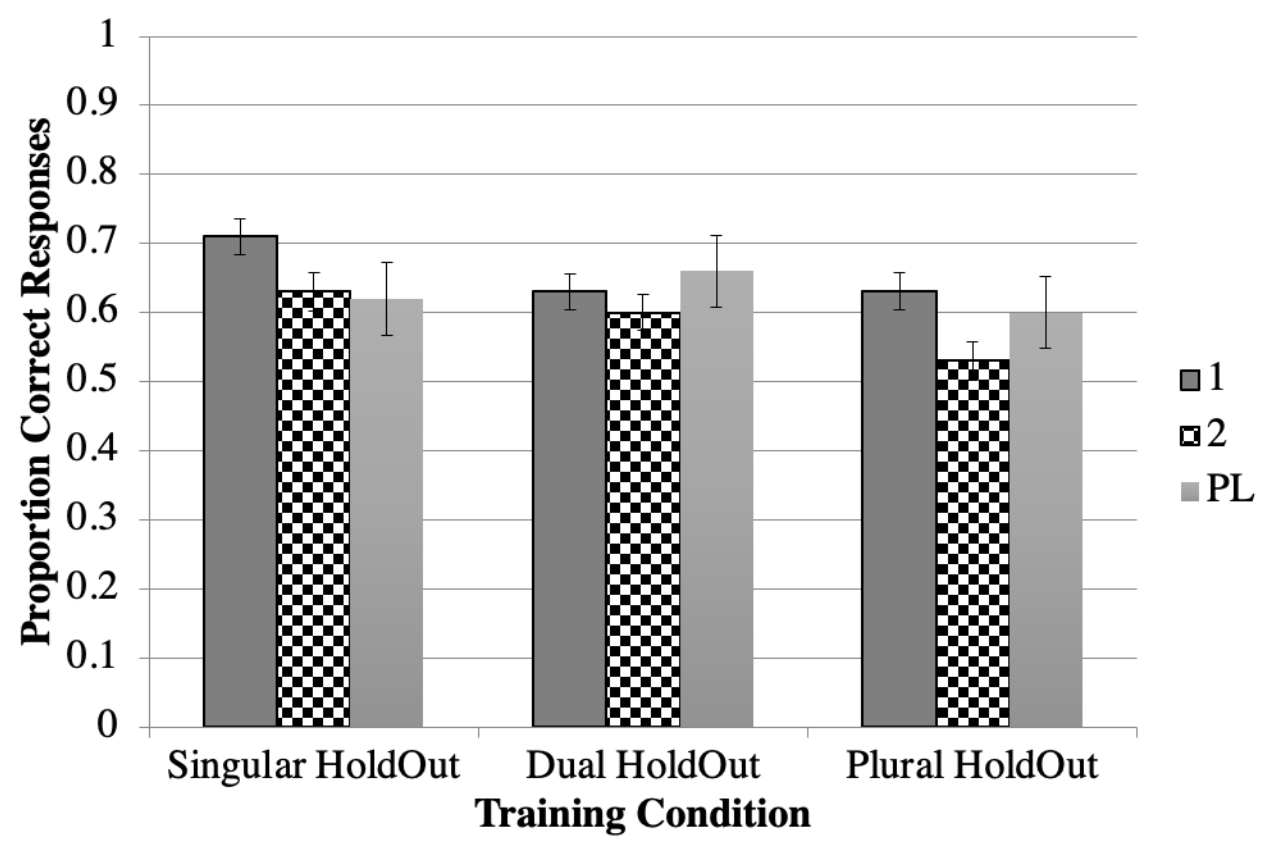

Figure 1: Experiment 2 Results: Familiar Affixes.

\section{Familiar Affixes: Dual Condition}

The model for the Dual condition showed a marginally significant intercept for dual (2) items, $\beta=0.54, \mathrm{SE}=0.32, \mathrm{z}=1.69, p=0.091$, suggesting that participants did not learn the dual form in a robust manner. Participants in the Dual condition were not significantly more likely to select the correct response to singular (1) items $\beta=0.16, \mathrm{SE}=0.26, \mathrm{z}=0.62, p=0.54$ or plural (PL) items $\beta=0.35, \mathrm{SE}=0.28, \mathrm{z}=1.26, p=0.21$, compared to dual (2) items. Unlike Experiment 1 there were not significantly more correct responses to dual items in either the Singular condition, $\beta=0.086, \mathrm{SE}=0.40, \mathrm{z}=0.22, p=0.83$, or the Plural condition, $\beta=-0.30, \mathrm{SE}=0.40, \mathrm{z}=-0.73, p$ $=0.46$. There were no significant condition by number interactions for the difference between dual (2) and singular (1) items between the Dual and Singular conditions, $\beta=0.24, \mathrm{SE}=0.36, \mathrm{z}=0.66$, $p=0.51$, or the Dual and Plural conditions, $\beta=0.30, \mathrm{SE}=0.30, \mathrm{z}=0.99, p=0.32$. There were no significant condition by number interactions for the difference between dual (2) and plural (PL) 
items between the Dual and both the Plural, $\beta=0.37, \mathrm{SE}=0.37, \mathrm{z}=1.02, p=0.31$, and between Singular and Dual conditions, $\beta=0.34, \mathrm{SE}=0.31, \mathrm{z}=1.13, p=0.26$.

\section{Familiar Affixes: Plural Condition}

The model for the Plural condition showed a significant intercept for plural (PL) items, $\beta$ $=0.83, \mathrm{SE}=0.33, \mathrm{z}=2.50, p=0.012$, suggesting that participants were able to learn the plural form. Participants in the Plural condition were not significantly more likely to select the correct response to singular (1) items $\beta=0.11, \mathrm{SE}=0.28, \mathrm{z}=0.41, p=0.68$, but were more likely to select the correct response to dual (2) items $\beta=0.58, \mathrm{SE}=0.28, \mathrm{z}=2.06, p=0.039$, compared to plural (PL) items. The Plural condition showed no significant differences between correct responses to plural (PL) items in the Dual condition, $\beta=0.044, \mathrm{SE}=0.44, \mathrm{z}=0.11, p=0.91$, or the Singular condition, $\beta=0.20, \mathrm{SE}=0.40, \mathrm{z}=0.50, p=0.62$. There was a marginally significant condition by number interaction in the difference between plural (PL) and dual (2) between the Plural and the Singular condition, $\beta=0.58, \mathrm{SE}=0.31, \mathrm{z}=1.89, p=0.059$. There was no significant condition by number interaction between the difference between plural (PL) and singular (1) items between the Plural and the Singular condition, $\beta=0.50, \mathrm{SE}=0.36, \mathrm{z}=1.36, p=0.17$, or between the Dual and Plural conditions, $\beta=0.052, \mathrm{SE}=0.31, \mathrm{z}=0.17, p=0.87$.

\section{Familiar Affixes: Singular Condition}

The model for the Singular condition showed a significant intercept for singular (1) items, $\beta=1.00, \mathrm{SE}=0.33, \mathrm{z}=3.07, p=0.0021$, suggesting that participants were able to learn the singular form. There were no significant differences in responses to singular (1) items compared to either plural (PL) $\beta=0.38, \mathrm{SE}=0.28, \mathrm{z}=1.37, p=0.17$, or dual (2) items $\beta=0.38, \mathrm{SE}=0.28, \mathrm{z}=1.35$, $p=0.18$, compared to plural (PL) items. There were no significant differences in responses to singular (1) items between the Singular condition and either the Dual condition, $\beta=0.30$, SE $=$ 
$0.41, \mathrm{z}=0.75, p=0.46$, or the Plural condition, $\beta=0.30, \mathrm{SE}=0.40, \mathrm{z}=0.73, p=0.46$. There was a significant condition by number interaction for the difference between singular (1) and dual (2) items between the Singular and the Plural condition, $\beta=0.087, \mathrm{SE}=0.31, \mathrm{z}=0.28, p=0.78$. The condition by number interaction for the difference between plural (PL) and singular (1) items between the Singular and the Dual condition was marginally significant, $\beta=0.55, \mathrm{SE}=0.31, \mathrm{z}=$ $1.73, p=0.081$.

\section{Held-Out Items}

The held-out items (masculine and feminine items) in each hold-out condition were analyzed separately to assess whether participants had inferred that these categories were syncretic with their number or their gender, with dual as the baseline. There were no significant differences between the Dual (mean $=0.52 \pm 0.067)$ and Singular (mean $=0.55 \pm 0.065)$ conditions for heldout items, $\beta=0.12$, $\mathrm{SE}=0.43, \mathrm{z}=0.28, p=0.78$, or between the Dual and Plural (mean $=0.49 \pm$ 0.066) conditions, $\beta=0.18, \mathrm{SE}=0.42, \mathrm{z}=0.43, p=0.67$. Thus, there were no overall differences across the conditions for how participants inferred syncretism should apply.

While the overall means for hold-out items are close to chance, participants might have shown a bias, for example, to select the syncretism by number when this reduced markedness (e.g., collapsed dual and plural). Participants were significantly more likely to select syncretism by gender when singular and dual collapsed (mean $=0.61 \pm 0.072)$ compared to when plural and dual collapsed $($ mean $=0.44 \pm 0.66), \beta=0.78, \mathrm{SE}=0.23, \mathrm{z}=3.37, p<0.001$, and were marginally more likely to select syncretism by gender when singular and dual were neutralized compared to when plural and singular $($ mean $=0.52 \pm 0.061)$ were neutralized, $\beta=0.42, \mathrm{SE}=0.23, \mathrm{z}=1.87, p$ $=0.062$. There was a significant intercept for singular-dual neutralization, $\beta=0.53, \mathrm{SE}=0.20, \mathrm{z}$ 
$=2.67, p=0.0072$, but not for singular-plural neutralization, $\beta=0.11, \mathrm{SE}=0.17, \mathrm{z}=0.63, p=$ 0.53, or for dual-plural neutralization, $\beta=-0.42$, $\mathrm{SE}=0.18, \mathrm{z}=-1.40, p=0.16$.

\section{Discussion}

Participants were successful at learning the neuter forms in the Singular and Plural condition, and marginally so in the Dual condition. This suggests that the failure to learn the syncretic affixes in Experiment 1 was not due to fewer overall numbers of items in the category, but fewer numbers from each specific category (e.g., masculine-singular in the Singular condition). However, there did appear to be a bias against the dual forms in Experiment 2, which emerged in the Dual and the Plural Hold-Out conditions. This is to be expected, as participants were English speakers with little prior knowledge of the dual.

In Experiment 2, the syncretic forms were always neuter, and thus participants had no information about the masculine or feminine in these forms. At test, participants had to choose between whether the non-neuter items were syncretic with the neuter forms (by gender, selecting the /-go/ affix) or if the non-neuter forms were syncretic with another number (syncretic by number). Participants showed a bias for syncretism across gender only when the alternative was to collapse singular and dual, which falls in line with expectations about syncretism for number. However, the tendency to collapse dual with plural was not statistically significant. This could be because syncretism for non-singular forms are often syncretic across gender, but syncretism for number often applies between dual and plural (Baerman et al., 2005). Thus, both types of syncretism fall in line with typological trends for syncretism in the dual.

\section{General Discussion}

While syncretism for gender is cross-linguistically more likely to apply across the nonsingular forms compared to singular forms (Baerman et al., 2005), learners in Experiment 1 
showed no bias towards syncretism in the dual or plural when frequency of syncretic items was low. Experiment 2 verified that the difficulty in learning the syncretic category in Experiment 1 was not due to low numbers of the overall category (e.g., 6 singular items and 18 each of dual and plural in the Singular condition). When the six items were all of the same gender (neuter), participants were able to learn the singular and the plural, and were marginal for the dual. This suggests that if syncretism is useful for learning low frequency categories, there needs to be enough information in each individual category for them to be learned.

In previous research, when the frequency of each category was equal, regardless of form, there was a benefit to having syncretism in the dual, the marked category (Finley, in press). In the present study, the frequency of the forms was matched. This meant that the syncretic category was less frequent; there were six forms for each ending, but only six syncretic forms (in the Singular condition), but 18 in each of the other categories. This lower frequency of items overall made learning the syncretic category harder, and participants showed no bias towards the dual in this case. If anything, participants assumed that the dual and the plural categories were syncretic, as they are in English, the dominant language of the participants in this study.

The present results have important consequences for theories of learning morphology, particularly complex morphological systems where semantic and featural markedness intersect with frequency. Previous research has suggested that syncretic categories carry more information, and can thus reduce processing time, and that there is a correlation between frequency of a category and its featural complexity (Parker, 2016). If learners are unable to discover the meanings for low frequency affixes or categories, then it raises the question of what happens to these categories. Either they simply take longer to learn, or over time, the language may combine with other categories, and the distinction may be lost. 


\section{Limitations and Suggestions for Future Research}

Because the results of the present study are essentially a null result, there are several limitations to be noted, many of which could be addressed in future research. Because participants in Experiment 1 failed to learn the syncretic categories across all conditions, it is difficult to make any specific claims related to learnability and typology. It is clear that low frequency of syncretic items in the present study hindered the learnability of these categories, but it is possible that a different experimental design might have yielded learnability in Experiment 1. One possibility is to reduce the complexity of the design. In the present study there was a 3 (gender) by 3 (number) system, but many languages have simpler systems, such as 2 (gender) x 2 (number). A replication of the present study with a simpler design could yield more consistent results. Another way to simplify the language could be to reduce the number of animal stem names that the participants were required to learn, and then increase the number of repetitions. Other possible ways to induce learning in the present study might be to simply increase the exposure phase or provide more direct feedback to learners as they are exposed to the language (e.g., have multiple tests on the words after shorter exposure, with feedback). Future research could work to clarify this process by addressing questions of how much data is required to learn a low-frequency category, and how inferences about low-frequency categories might change over time, and whether that contributes to the cross-linguistic typology of syncretism.

Another possible way to address the questions raised in the present study would be to test whether the typological trends for syncretism across marked categories emerge as a result of frequency, or as a result of their inherent markedness, would be to use iterated learning models in order to control for frequency and category markedness across generations of speakers (Kirby et al., 2008; Reali \& Griffiths, 2010; Smith et al., 2003; Smith \& Wonnacott, 2010; Vihman et al., 
2018). Previous research has suggested that some learning biases do not emerge well in a single learning experiment, and that biases are more likely to reveal themselves over multiple generations of speakers (Reali \& Griffiths, 2010). One reason for this is that in a laboratory setting, the language needs to be simple enough, with enough data in order to show learning results, but when there is enough data, differences between conditions become masked. This may be an issue with Experiment 1 . There was not enough data to learn the syncretic forms, but simplification, more data, or increased training, may mask any differences between conditions. For example, in Experiment 2, when there was just one gender category in the low frequency number, participants showed learning of the category (though only marginally for dual).

An iterative learning experiment could help to model how syncretism might emerge over time. Thus, rather than have the language at the 'end state' where the low frequency categories are all syncretic, an iterative learning model could start with no syncretism in any category, and see whether syncretism is more likely to occur over generations based on semantic markedness, frequency, or an interaction between the two. This question could also be tested by comparing a language with syncretism to one without (assuming that the low frequency category is learnable to some degree).

In addition to issues with frequency and learnability, the gender categories that were used in the present study were arbitrary, and ambiguous between a noun class system and a biosocial gender system. Because each stem could be assigned with each gender, and the affixes were not explicitly used as agreement markers, the morphological system in the present study may be more like an inflectional class system than a gender system, which may have implications for connecting the typology of syncretism across gender systems with the present study. Future research could 
address issues related to the differences between learning noun classes and inflectional classes as they relate to syncretism.

\section{Conclusions}

This paper presented two experiments exploring the learnability of syncretic forms when the tokens of items were matched to other tokens in the language, thereby significantly decreasing the total number of words in the language from the syncretic category. Participants failed to learn the syncretic category in Experiment 1, but were able to learn the singular and plural in Experiment 2 , when the low frequency category was always neuter (and marginally so for dual). This suggests that learnability of syncretism requires greater frequency of the syncretic forms in order for there to be a benefit from syncretism.

\section{References}

Aalberse, S. (2009). Inflectional economy and politeness: Morphology-internal and morphologyexternal factors in the loss of second person marking in Dutch. LOT.

Baerman, M., Brown, D., \& Corbett, G. G. (2005). The syntax-morphology interface: A study of syncretism. Cambridge University Press.

Bates, D., Mächler, M., Bolker, B., \& Walker, S. (2015). Fitting linear mixed-effects models using $\{1 \mathrm{me} 4\}$. Journal of Statistical Software, 67, 1-48. https://doi.org/10.18637/jss.v067.i01

Corbett, G. G. (1991). Gender. Cambridge University Press.

Corbett, G. G. (2012). Gender. Cambridge University Press.

FindingFive Team. (2019). FindingFive: A web platform for creating, running, and managing your studies in one place. FindingFive Corporation (nonprofit),. https://www.findingfive.com

Finley, S. (n.d.). Learning complex morphological patterns. In Proceedings of the Morphology, 
Typology, and Linguistic Cognition Workshop.

Finley, S., \& Wiemers, E. (2015). Phonological and semantic consistency as cues for learning morphological systems. In U. Steindl (Ed.), Proceedings of the 32nd West Coast Conference on Formal Linguistics (pp. 11-20). Cascadilla Proceedings Project.

Harmon, Z., \& Kapatsinski, V. (2017). Putting old tools to novel uses: The role of form accessibility in semantic extension. Cognitive Psychology, 98, 22-44. https://doi.org/10.1016/j.cogpsych.2017.08.002

Haspelmath, M. (2006). Against markedness (and what to replace it with). Journal of Linguistics, 42(1), 25-70. https://doi.org/10.1017/S0022226705003683

Kirby, S., Cornish, H., \& Smith, K. (2008). Cumulative cultural evolution in the laboratory: An experimental approach to the origins of structure in human language. Proceedings of the National Academy of Sciences of the United States of America, 105(31), 10681-10686. https://doi.org/10.1073/pnas.0707835105

Lee, N. (2020). Learning (im)possible number syncretisms: Investigating innate featural representations.

Müller, G. (2007). Notes on paradigm economy. Morphology, 17(1), 1-38. https://doi.org/10.1007/s11525-007-9114-x

Nevins, A. I. (2006). Dual is still more marked than plural. Harvard Working Papers in Linguistics, 12.

Nevins, A. I. (2011). Marked targets versus marked triggers and impoverishment of the dual. Linguistic Inquiry, 42(3), 413-444. https://doi.org/10.1162/LING_a_00052

Parker, J. (2016). Inflectional complexity and cognitive processing: An experimental and corpusbased investigation of Russian nouns. Doctoral Dissertation, The Ohio State University. 
Pertsova, K. (2011). Grounding systematic syncretism in learning. Linguistic Inquiry, 42(2), 225266. https://doi.org/10.1162/LING_a_00041

R Development Core Team, R. (2018). R: A language and environment for statistical computing. In R. D. C. Team (Ed.), R Foundation for Statistical Computing (Vol. 1, Issue 2.11.1, p. 409). R Foundation for Statistical Computing. https://doi.org/10.1007/978-3-540-74686-7

Reali, F., \& Griffiths, T. L. (2010). Words as alleles: connecting language evolution with Bayesian learners to models of genetic drift. October 2009, 429-436. https://doi.org/10.1098/rspb.2009.1513

RStudio Team. (2020). RStudio: Integrated Development for R. 2020. http://www.rstudio.com/

Smith, K., Kirby, S., \& Brighton, H. (2003). Iterated Learning: A Framework for the Emergence of Language. Artificial Life, 9(4), 371-386. https://doi.org/10.1162/106454603322694825

Smith, K., \& Wonnacott, E. (2010). Eliminating unpredictable variation through iterated learning. Cognition, 116(3), 444-449. https://doi.org/10.1016/j.cognition.2010.06.004

Vihman, V. A., Nelson, D., \& Kirby, S. (2018). Animacy distinctions arise from Iierated learning. Open Linguistics, 4(1), 552-565. https://doi.org/10.1515/opli-2018-0027 